\title{
Desenvolvimento e caracterização físico-química de macarrão com substituição parcial da farinha de trigo por farinha de polpa de baru
}

\author{
Development and physicochemical characterization of noodles with partial replacement of wheat
}

\author{
flour by baru pulp flour
}

Desarrollo y caracterización fisicoquímica de los fideos con sustitución parcial de harina de trigo

por harina de pulpa barú

Recebido: 02/10/2021 | Revisado: 08/10/2021 | Aceito: 14/10/2021 | Publicado: 17/10/2021

\author{
Gabriella Gonçalves Borges Antunes \\ ORCID: https://orcid.org/0000-0002-6769-5666 \\ Instituto Federal de Goiás Campus Goiânia, Brasil \\ E-mail: gabriellaborges.antunes@gmail.com \\ Thairyne Naruan Alves Pereira \\ ORCID: https://orcid.org/0000-0002-3640-0810 \\ Universidade Federal de Goiás, Brasil \\ E-mail: thairynenaruan@gmail.com \\ Joema Rodrigues Cardoso Santos \\ ORCID: https://orcid.org/0000-0001-7314-5225 \\ Universidade Federal de Goiás, Brasil \\ E-mail: eng.quim.joema@gmail.com \\ Marcos dos Reis Vargas \\ ORCID: https://orcid.org/0000-0002-1178-8839 \\ Instituto Federal de Goiás Campus Goiânia, Brasil \\ E-mail: marcos.vargas@ifg.edu.br
}

\begin{abstract}
Resumo
A flora do bioma Cerrado é constituída por diversas frutíferas, as quais apresentam potencial biotecnológico de grande importância. A aplicação dessas no desenvolvimento de novos produtos contribui para valorização das espécies regionais. Dentre os vegetais característicos da região, destaca-se o baru (Dipteryx alata Vog.), do qual a amêndoa é amplamente utilizada; porém, a polpa rica em fibras, é comumente descartada. Portanto, o objetivo do presente trabalho foi desenvolver macarrões com substituições de 10 e $20 \%$ da farinha de trigo pela farinha de polpa de baru. Foram realizadas análises físico-químicas, microbiológicas e sensoriais. Os resultados demonstraram que as massas alimentícias com farinha de polpa de baru podem ser classificadas como alimentos com alto teor de fibra alimentar e ainda, apresentaram menor teor de carboidratos que a amostra controle (100\% de farinha de trigo). Os testes sensoriais resultaram em escores médios entre 5 e 6, de uma escala de 9 pontos, para os macarrões. Logo, os resultados apresentados possibilitam a ampliação de aplicação da polpa de baru na indústria alimentícia.
\end{abstract}

Palavras-chave: Baru; Polpa; Macarrão.

\begin{abstract}
The flora of the Cerrado biome is made up of several fruit trees, which have a very impor-tant biotechnological potential. The application of these in the development of new products contributes to the appreciation of regional species. Among the characteristic plants of the region, the baru (Dipteryx alata Vog.), Of which the almond is widely used, stands out; ho-wever, the fiber-rich pulp is commonly discarded. Therefore, the objective of the present work was to develop noodles with substitutions of 10 and $20 \%$ of the wheat flour for the pulp flour of baru. Physicochemical, microbiological and sensorial analyzes were performed. The results showed that pasta with baru pulp flour can be classified as foods with high dietary fiber content and also had a lower carbohydrate content than the control sample (100\% wheat flour). Sensory tests resulted in mean scores of 5 to 6 on a 9-point scale for noodles. Therefore, the presented results allow the expansion of the application of the pulp of baru in the food industry.
\end{abstract}

Keywords: Baru; Pulp; Pasta.

\section{Resumen}

La flora del bioma del Cerrado está formada por varios árboles frutales, los cuales tienen un potencial biotecnológico de gran importancia. La aplicación de estos en el desarrollo de nuevos productos contribuye a la mejora de especies regionales. Entre las hortalizas características de la región, destaca el baru (Dipteryx alata Vog.), De la que se utiliza mucho la almendra; sin embargo, la pulpa rica en fibra se descarta comúnmente. Por lo tanto, el objetivo del presente trabajo fue desarrollar fideos con 10 y $20 \%$ de sustituciones de harina de trigo por harina de pulpa de barú. Se realizaron 
análisis fisicoquímicos, microbiológicos y sensoriales. Los resultados mostraron que la pasta con harina de pulpa de barú se puede clasificar como alimentos con un alto contenido de fibra dietética y además tenía un contenido de carbohidratos menor que la muestra control (100\% harina de trigo). Las pruebas sensoriales dieron como resultado puntuaciones medias entre 5 y 6 , en una escala de 9 puntos, para los fideos. Por tanto, los resultados presentados permiten ampliar la aplicación de la pulpa de baru en la industria alimentaria.

Palabras clave: Barú; Pulpa; Fideo.

\section{Introdução}

O macarrão, também denominado de massa alimentícia, é o produto não fermentado, obtido pelo amassamento da farinha de trigo, da semolina ou da sêmola de trigo com água, adicionado ou não de outras substâncias (Brasil, 1978). Atualmente, tem-se observado uma tendência à substituição parcial de farinha de trigo nas formulações por farinhas de diversos frutos (Neto et al., 2016; Gabriel Pereira et al., 2017; Neto et al., 2013; Pietro 2016). A substituição é justificada pela disponibilidade de macarrões desenvolvidos a partir de formulações de baixo valor nutricional, com destaque à deficiência em fibras. Assim, tais deficiências nutricionais podem ser compensadas pela adição de outros ingredientes às massas alimentícias, que aliado ao seu baixo custo, pode torna-los um item importante na alimentação (Neto et al., 2016).

Dentre os frutos disponíveis para substituição parcial, tem-se o baru (Dipteryx alata Vogel), especificamente a utilização da sua polpa para tal finalidade. O fruto do baru é do tipo drupa, ovóide, levemente achatado e de coloração marrom, com uma única semente comestível, a amêndoa de baru; apresenta diâmetro maior entre $5 \mathrm{a} 7 \mathrm{~cm}$ e o diâmetro menor de 3 a $5 \mathrm{~cm}$, com peso entre 26 a 40g (Vera et al., 2009). A amêndoa caracteriza-se por ser rica em lipídeos (41mg/ 100g), proteínas (26mg/100g), alto teor de fibra total $(11 \mathrm{mg} / 100 \mathrm{~g})$ e minerais $(3 \mathrm{mg} / 100 \mathrm{~g})$ (Takemoto et al., 2001). A polpa é do tipo carnosa, apresentando aproximadamente 300kcal em 100g. Dentre os carboidratos, há predomínio de amido, fibras insolúveis e açúcares (Sano et al. 2004). Porém, essa é pouca inserida na alimentação humana apesar de inúmeras possibilidades de aplicação alimentícia após seu processamento (Rocha \& Cardoso - Santiago, 2009). Em termos de rendimento, o barueiro (árvore do baru) produz de 2.000 a 6.000 frutos por planta (Cruz et al., 2011). Tais frutos são compostos por 30\% de polpa, 5\% de amêndoa e $65 \%$ de endocarpo (Carraza \& Ávila, 2010).

O barueiro ocorre geralmente nas áreas férteis do Cerrado (http://floradobrasil.jbrj.gov.br/, recuperado em 01 de outubro, 2021). O Cerrado é o segundo maior bioma brasileiro (ficando atrás apenas da Amazônia), ocupando quase toda a área do Brasil central, com aproximadamente 2 milhões de km2 (Klink \& Machado, 2005). Ocorre nos estados de São Paulo, Minas gerais, Goiás, Mato Grosso, Mato Grosso do Sul, Tocantins, Bahia, Maranhão, Piauí e Distrito Federal; além disso, incorpora ainda partes do território de países vizinhos como Bolívia e Paraguai (Cavalcanti et al., 2012). Apresentando uma grande variedade de espécies, no tocante à fauna e flora, o cerrado é composto por mais de 10.000 espécies de plantas (Dijalma Silva et al., 2001; Scariot et al., 2005).

Percebe-se que o desenvolvimento de novos produtos a partir de matrizes vegetais nativas da região constitui uma valorização que contribui para a preservação de tais espécies, uma vez que o Cerrado, depois da Mata Atlântica é o ecossistema que mais sofreu alterações com a ocupação humana (http://www.wwf.org.br/natureza_brasileira/questoes_ambi entais/biomas/bioma_cerrado/mapa_bioma_cerrado, recuperado em 01 de outubro, 2021). Ainda, a incorporação da espécie citada no desenvolvimento de novos produtos incentiva o desenvolvimento regional sustentável, fomentando a agricultura familiar, caracterizada pela produção em pequena escala (Carrazza, 2010).

Portanto, o objetivo do trabalho foi desenvolver uma massa alimentícia do tipo macarrão, a partir de formulações com substituição parcial da farinha de trigo pela farinha de polpa de baru, propondo-se obter um produto fonte de fibras. 


\section{Metodologia}

O presente trabalho caracteriza - se como documentação direta, uma vez que o levantamento de dados ocorreu no próprio local onde os fenômenos ocorrem, conforme apontado por Marconi \& Lakatos (2017). Para obtenção dos dados, utilizou - se a pesquisa laboratorial, a qual descreve e analisa o que ocorrerá em situações controladas, com o objetivo adequado ao que se pretende alcançar (Marconi \& Lakatos, 2017).

Ainda, o desenvolvimento da pesquisa foi pautado pela utilização de métodos quantitativos, pois realizou -se a coleta de dados por meio do uso de medições e grandezas, obtendo - se por meio da metrologia, números (resultados) com suas respectivas unidades (Adriana Pereira et al., 2018). Como suporte metodológico, utilizou - se o trabalho desenvolvido por Pietro (2016), para direcionamento do desenvolvimento de formulações e escolha das análises realizadas.

Os frutos da espécie Dipteryx alata Vogel foram adquiridos em outubro de 2017 de um pequeno produtor, cuja plantação está localizada no município de Rio Verde (Estado de Goiás, Brasil). Logo após a aquisição dos frutos, esses foram lavados em água corrente e, na sequência, foi realizada a separação entre a castanha de baru e a polpa de baru juntamente com o endocarpo.

O material permaneceu por dois meses sob refrigeração a $-4^{\circ} \mathrm{C}$. Para o processamento da farinha, a polpa e o endocarpo foram submetidos à secagem a $60^{\circ} \mathrm{C}$ por $12 \mathrm{~h}$ e $30 \mathrm{~min}$ em forno elétrico. Como parâmetros para determinação do tempo suficiente de secagem, foram utilizadas a observação da cor e da textura apresentada pela polpa e endocarpo de baru (Pietro, 2016).

A moagem da polpa e do endocarpo de baru foi realizada inicialmente em liquidificador industrial e na sequência em moinho de facas tipo willye. A análise granulométrica da farinha de polpa de baru foi realizada a partir da adaptação de método AACC 66-20 (American Association of Cereal Chemistry) (AACC, 1995a), utilizando-se peneiras granulométricas taradas

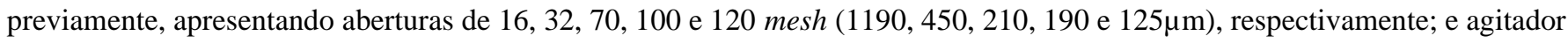
eletromagnético de peneiras. Para produção da massa alimentícia, optou-se por utilizar a porção retida na peneira de 70 mesh, uma vez que para produção de macarrão utiliza-se farinhas com granulometria variando entre 60 e 70 mesh (Guerreiro, 2006).

As massas alimentícias foram elaboradas na planta didática da Escola SENAI Vila Canaã, localizada no município de Goiânia -GO. Foram realizadas três formulações, sendo uma de controle (contendo somente farinha de trigo tipo 1); e duas formulações com farinha de trigo tipo 1 e farinha de polpa de baru nas proporções de 90:10 e 80:20, respectivamente. As três formulações desenvolvidas estão representadas na Tabela 1.

Tabela 1: Formulações das massas alimentícias.

\begin{tabular}{cccc}
\hline Formulações & FT & F1 & F2 \\
Farinha de trigo & $100 \%$ & $90 \%$ & $80 \%$ \\
Farinha de polpa de baru & - & $10 \%$ & $20 \%$ \\
Ovo & $55 \%$ & $55 \%$ & $55 \%$ \\
\hline
\end{tabular}

Legenda: FT - amostra controle (100\% de farinha de trigo); F1 - amostra com 10\% da farinha de polpa de baru; F2 amostra com $20 \%$ da farinha de polpa de baru.

Fonte: Antunes et al.

Os ingredientes foram misturados em masseira; a mistura resultante foi transferida para um canhão extrusor, para obterse macarrão extrusado. Durante moldagem ou trefilação, a massa foi prensada por uma trefila de bucatini e o corte feito manualmente, com tamanho padronizado de $10 \mathrm{~cm}$. Após a trefilação e o seccionamento, os espaguetes foram secos a $60^{\circ} \mathrm{C}$ durante 1 hora em estufa com circulação e renovação de ar. As massas alimentícias secas foram empacotadas e armazenadas a 
$6^{\circ} \mathrm{C}$ para posterior análises, cocção e testes sensoriais.

\subsection{Análises microbiológicas}

Após o desenvolvimento das massas alimentícias, reservou-se uma quantidade de macarrão para análise microbiológica. As análises microbiológicas realizadas compreendem: Salmonella sp (ISO, 2002; AOAC, 1997); Bacilus cereus (ISO, 2004); coliformes a $45^{\circ} \mathrm{C}$ (ISO, 2004); e Staphylococcus aureus (APHA, 2001). Tais parâmetros de análise microbiológica foram estabelecidos conforme preconiza a RDC nº 12 (Brasil, 2001).

\subsection{Análises físico-químicas}

A determinação da composição centesimal da farinha de polpa do baru e das formulações desenvolvidas foi realizada no laboratório didático da Escola SENAI Vila Canaã e no laboratório de Análises Físico-Químicas do Instituto Federal de Goiás Campus Goiânia. A umidade foi analisada em estufa de secagem com circulação de ar à $105^{\circ} \mathrm{C}$ até obtenção de peso constante (método AOAC nº 925.10 - Association Of Official Analytical Chemists), e o resíduo mineral fixo (cinzas) por incineração em mufla a $550^{\circ} \mathrm{C}$ (método $\mathrm{AOAC} \mathrm{n}^{\circ}$ 923.03) (AOAC, 1997). Os teores proteicos foram obtidos por meio da análise de nitrogênio total por Kjeldahl (método NTK), sendo utilizado o fator adequado para a conversão do nitrogênio em proteína bruta para consumo (método AOAC n 960.52) (AOAC, 1997). A fração de fibra alimentar total foi analisada pelo método gravimétricoenzimático estabelecido pela Association of Analytical Communities - AOAC, adaptado pela Embrapa Agroindústria de Alimentos (método AOAC n ${ }^{\circ}$ 985.29) (Freitas et al., 2011). Os lipídios totais foram extraídos pelo método de Bligh-Dyer (método AOAC $n^{\circ}$ 920.39C) (AOAC, 1997). Os carboidratos disponíveis foram obtidos por diferença, subtraindo-se de cem os valores obtidos de umidade, cinzas, proteínas, lipídios e fibra alimentar total, de acordo com o estipulado na Resolução RDC no 360 de 2003 que trata sobre rotulagem de alimentos (Brasil, 2003). A acidez e o pH foram determinados conforme estabelecido pelo Instituto Adolfo Lutz (IAL, 2008). O valor energético total (VET) foi estimado considerando-se os fatores de conversão de Atwater, atribuindo 4kcal g-1 para proteínas e carboidratos e 9kcal g-1 para lipídeos (Atwater \& Woods, 1986).

\subsection{Testes de qualidade das massas}

Os testes de qualidade das massas compreenderam:

Tempo de cozimento: O tempo de cozimento foi determinado a partir da cocção de $10 \mathrm{~g}$ de amostra de macarrão em $140 \mathrm{~mL}$ de água destilada em ebulição. O ponto ideal de cozimento foi obtido a partir da compressão de amostras do macarrão cozido, a cada 30 segundos, entre duas lâminas de vidro até o desaparecimento do eixo central (AACCb, 1995).

Aumento da massa do produto cozido: $\mathrm{O}$ aumento da massa após cozimento foi determinado pela pesagem da amostra antes e após a cocção, utilizando-se o tempo de cozimento ideal de cada amostra. O valor do aumento da massa é a razão entre a massa cozida e a massa crua (10g), expresso em porcentagem (\%) (AACCb, 1995).

Perda sólidos na água de cozimento: A perda de sólidos na água de cozimento foi determinada pela evaporação de $25 \mathrm{~mL}$ de amostra em estufa a $105^{\circ} \mathrm{C}$, até peso constante (AACCb, 1995). A porcentagem de sólidos perdidos foi calculada utilizando-se a equação representada na Figura 1: 
Figura 1: Equação para determinação de porcentagem de sólidos perdidos.

$$
\% \text { sólidos perdidos }=\frac{\text { Peso do resíduo evaporado }(g) \times \text { Volume de água de cozimento }(m L) \times 100}{\text { Peso da amostras }(g) \times \text { Volume da alíquota }(m L)}
$$

Fonte: AACC (1995b).

Onde o volume de água de cozimento foi medido com proveta de capacidade 200,0mL, sendo $140,0 \mathrm{~mL}$ de água e o volume da alíquota de $25,0 \mathrm{~mL}$.

\subsection{Cocção}

As massas foram cozidas em água em ebulição, contendo $4 \%$ de cloreto de sódio. O tempo de cozimento empregado foi equivalente ao tempo obtido no teste de cozimento.

\subsection{Análise sensorial}

A análise sensorial foi realizada com 30 provadores não treinados, acadêmicos, de uma faixa etária de 18 a 30 anos, no Laboratório Gastronômico do Instituto Federal de Goiás Campus Goiânia. Aplicou-se o teste de comparação pareada, no qual o provador deveria assinalar qual amostra preferia às outras. Aplicou-se também o teste de aceitação por escala hedônica de nove pontos, variando de 9 - gostei muitíssimo a 1 - desgostei muitíssimo (Faria \& Yotsuyanagi, 2002). Além dos testes anteriores, aplicou-se o teste de intenção de compra, variando de 5 - certamente compraria a 1- certamente não compraria (Faria \& Yotsuyanagi, 2002).

\subsection{Tratamento estatístico dos dados}

Avaliou-se os resultados obtidos pelos métodos de análise de variância (ANOVA) e as médias foram comparadas pelo teste de Tukey a 5\% de significância (Teixeira et al., 1987).

\section{Resultados e Discussão}

\subsection{Separação, moagem e granulometria}

Após a etapa de higienização, realizou-se a separação entre polpa e endocarpo e, amêndoa de polpa de baru. Obteve-se uma extração de $8 \%$ da amêndoa de baru e de $92 \%$ para a extração da polpa e do endocarpo de baru. Segundo Martins (2010), tais rendimentos de extração indicam que o beneficiamento do baru apresenta elevados índices de geração de resíduos sólidos. O mesmo autor obteve uma extração da amêndoa variando entre 7,4 a 8,8\%, enquanto que para a polpa e para o endocarpo, um rendimento entre 88 a 95\%. Compreende-se como beneficiamento a separação da amêndoa do baru das demais estruturas do fruto (Carraza \& Ávila, 2010).

$\mathrm{Na}$ etapa de separação do endocarpo do mesocarpo, obteve-se um rendimento de $45 \%$, uma vez que em tal etapa separou-se a polpa do endocarpo do baru.

Na Figura 2, observa-se o gráfico representando os resultados de peneiramento. Ressalta-se que as porcentagens apresentadas referem-se à porcentagem média retida das farinhas em cada peneira. 
Figura 2: Distribuição granulométrica.

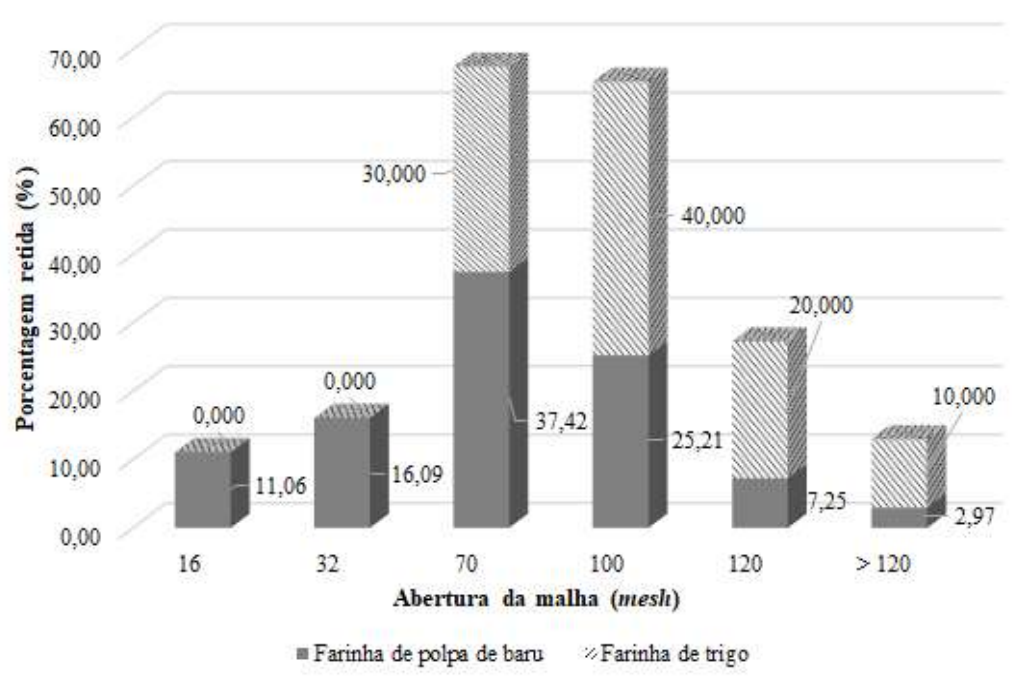

Fonte: Antunes et al.

Observou-se que a granulometria da farinha de trigo tipo 1 é mais fina que a farinha de polpa de baru, uma vez que $30 \%$ da farinha tipo 1 ficou retida na peneira de 70 mesh (Figura 2), enquanto 37,42\% da farinha de polpa de baru ficou retida na peneira de mesma malha. Na literatura, o tamanho da partícula do alimento após a moagem é um fator determinante no preparo de massas alimentícias, uma vez que a uniformidade da granulometria na farinha resulta em melhor qualidade sensorial. Ainda, durante o cozimento o alimento absorve água de forma homogênea, resultando no cozimento uniforme da massa (Reginaldo Silva et al., 2009).

A partir do tratamento estatístico da análise granulométrica, observou-se que para a farinha de polpa de baru, há diferença significativa entre as porcentagens retidas nas peneiras de 70 e 120 mesh, a nível de 5\% de significância. O resultado é justificado pela variação entre o tamanho dos grânulos da farinha de polpa de baru, após a etapa de moagem. Em contrapartida, o mesmo não é observado para a farinha de trigo. Para o desenvolvimento das massas alimentícias, foram utilizadas as farinhas de 70 mesh.

\subsection{Análises físico-químicas da farinha de polpa de baru e das massas alimentícias}

Os resultados das análises físico-químicas da farinha de polpa de baru e das massas alimentícias desenvolvidas nesse trabalho podem ser observados na Tabela 2. 
Tabela 2: Resultados das análises físico-químicas.

\begin{tabular}{ccccc}
\hline Análises & FPB & FT & F1 & F2 \\
\hline Umidade (\%) & $11,300 \pm 1,200^{\mathrm{a}}$ & $4,100 \pm 0,153^{\mathrm{b}}$ & $6,223 \pm 0,204^{\mathrm{c}}$ & $7,400 \pm 0,104^{\mathrm{c}}$ \\
Cinzas (\%) & $4,975 \pm 0,638^{\mathrm{a}}$ & $2,185 \pm 0,856^{\mathrm{b}}$ & $4,135 \pm 0,147^{\mathrm{a}}$ & $3,595 \pm, 539^{\mathrm{c}}$ \\
Proteínas (\%) & $4,149 \pm 0,121^{\mathrm{a}}$ & $11,450 \pm 0,728^{\mathrm{b}}$ & $9,643 \pm 0,742^{\mathrm{c}}$ & $9,533 \pm 0,589^{\mathrm{d}}$ \\
Lipídeos (\%) & $5,250 \pm 0,120^{\mathrm{a}}$ & $2,460 \pm 0,782^{\mathrm{b}}$ & $3,160 \pm 0,495^{\mathrm{c}}$ & $3,270 \pm 0,783^{\mathrm{d}}$ \\
Carboidratos (\%) & $35,362 \pm 0,488^{\mathrm{a}}$ & $66,437 \pm 0,061^{\mathrm{b}}$ & $64,878 \pm 0,385^{\mathrm{c}}$ & $61,692 \pm 0,926^{\mathrm{d}}$ \\
$\begin{array}{c}\text { Fibra alimentar total } \\
(\%)\end{array}$ & 39,500 & 5,500 & 11,960 & 14,510 \\
$\begin{array}{c}\text { Fibra alimentar } \\
\text { solúvel (\%) }\end{array}$ & 0,6100 & 2,610 & & 12,020 \\
$\begin{array}{c}\text { Fibra alimentar } \\
\text { insolúvel (\%) }\end{array}$ & 38,890 & 2,890 & 9,660 & 2,490 \\
pH & $5,433 \pm 0,058^{\mathrm{a}}$ & $6,167 \pm 0,058^{\mathrm{b}}$ & $5,700 \pm 0,100^{\mathrm{c}}$ & $5,633 \pm 0,058^{\mathrm{d}}$ \\
$\begin{array}{c}\text { Acidez (mg 100 g } \\
\text { Valor energético } \\
\text { (kcal g-1) }\end{array}$ & $8,108 \pm 0,125^{\mathrm{a}}$ & $3,051 \pm 0,513^{\mathrm{b}}$ & $2,227 \pm 0,027^{\mathrm{c}}$ & $5,025 \pm 0,824^{\mathrm{d}}$ \\
\hline
\end{tabular}

Legenda: FPB - farinha de polpa de baru; FT - amostra controle (100\% de farinha de trigo); F1 - amostra com 10\% da farinha de polpa de baru; F2 - amostra com $20 \%$ da farinha de polpa de baru.

$\mathrm{a}, \mathrm{b}, \mathrm{c}$ e d - médias seguidas de mesma letra não apresentam diferença significativa entre si pelo teste de Tukey a $5 \%$ de probabilidade.

Fonte: Antunes et al.

A partir dos resultados apresentados na Tabela 2, pode ser observado que o teor de umidade apresentado pela farinha de polpa de baru se encontra dentro do limite estabelecido pela RDC n 12 (Brasil, 1978), a qual enuncia que o limite máximo é de $14 \%$. A mesma observação pode ser estendida à umidade das massas alimentícias, uma vez que a RDC $\mathrm{n}^{\circ} 93$ estabelece $\mathrm{o}$ limite máximo de 13,0\% para massa alimentícia seca, a qual é caracterizada por ser submetida ao processo de secagem (Brasil, 2000). Como as massas alimentícias desenvolvidas no presente trabalho foram secas após trefilação, são classificadas como massas secas. Os mesmos resultados não foram encontrados em alguns trabalhos que compõem a literatura (Neto et al., 2016; Gabriel Pereira et al., 2017; Pietro, 2016), nos quais se observa que os teores de umidade variaram de 28 a $35 \%$. A diferença entre os resultados encontrados e os resultados obtidos por demais autores pode ser justificada pelo tipo de massa elaborada nos trabalhos, a qual apresenta característica de uma massa alimentícia fresca, que pode ou não ser submetida ao processo de secagem parcial, de forma que o produto final apresente umidade máxima de 35,0\% (Brasil, 2000).

Em relação aos teores de cinzas, observou-se que a farinha de polpa de baru apresenta teor de 4,975\%, dos quais segundo Daiana Silva (2017), estão presentes potássio, cálcio, fósforo, magnésio como macrominerais. O mesmo autor destaca que em farinhas de polpa de baru, o macromineral potássio é o que se apresenta em maior concentração (Daiana Silva, 2017). Ainda, observa-se que as massas alimentícias com farinha de polpa de baru apresentam teores de cinzas maiores que o macarrão controle (100\% de farinha de trigo), conferindo assim melhor conteúdo nutricional ao alimento.

Os números encontrados para proteína se aproximam aos determinados por Pietro (2016) para as massas alimentícias com 10 e $20 \%$ de substituição parcial da farinha de trigo por polpa de baru, trabalho no qual observou-se 11,86 e 10,99\% de proteínas, respectivamente. Ainda, ressalta-se que consumindo as massas com substituição parcial de 10 e $20 \%$ supre-se parte da necessidade diária de ingestão protéica, a qual é necessária para o crescimento, conservação e reparação de órgãos, tecidos e células (Neto et al., 2016). 
Para os valores de lipídeos, nota-se que as massas alimentícias desenvolvidas com farinha de polpa de baru apresentam teor acima de 3\%. Assim, de acordo com a RDC 54/ 2012, essas não podem ser consideradas como alimentos de baixo teor lipídico, uma vez que tal classificação se restringe a alimentos com no máximo 3\% de lipídeos (Brasil, 2012).

A análise de teor de carboidratos mostrou que todos os macarrões desenvolvidos apresentaram percentuais variando de 66 a 60\%, aproximadamente, para cada 100 gramas do produto. Segundo a OMS (2003), a quantidade mínima de carboidrato necessária para promover glicose às células cerebrais é estimada em 130g/ dia para adultos com idade superior a 19 anos. Assim o consumo de 100 gramas das massas desenvolvidas com farinha de polpa de baru fornece, aproximadamente, 50\% das necessidades diárias. $\mathrm{O}$ valor de carboidratos obtido para a farinha de polpa de baru corrobora com o valor encontrado por Mendonça (2008), que foi de 58,04\%. Incluso nesses 50\% de carboidratos estão o amido (componente majoritário) e açúcares (Sano et al., 2004).

Os teores de fibra alimentar total apresentado pelas massas alimentícias estão acima de 3\%. De acordo com a Resolução n 54 da ANVISA/ 2012 (Brasil, 2012), um alimento sólido é considerado como fonte de fibra alimentar se apresentar em sua composição no mínimo 3\%; caso apresente no mínimo $6 \%$ de fibra alimentar total pode ser classificado como um alimento com alto conteúdo de fibra alimentar. Portanto, o macarrão desenvolvido com 100\% de farinha de trigo é classificado como um alimento fonte de fibra alimentar, enquanto que as massas alimentícias com substituição parcial da farinha de trigo podem ser classificadas como alimentos com alto conteúdo de fibra alimentar. Ressalta-se que as fibras solúveis são responsáveis pelo aumento da viscosidade do conteúdo gastrointestinal, retardando o esvaziamento e a difusão de nutrientes, além de tornarem mais lenta a absorção de glicose e retardarem a digestão do amido (IAL, 2008). Em contrapartida, as fibras insolúveis, como a lignina, celulose e algumas hemiceluloses, aceleram o trânsito intestinal, aumentam o peso das fezes e contribuem para a redução do risco de alguns males do cólon (Neto, 2012).

O pH apresentado pela farinha de polpa de baru corrobora com o resultado obtido por Daiana Silva (2017), onde a média de $\mathrm{pH}$ para a farinha de endocarpo e mesocarpo de baru seca a $60^{\circ} \mathrm{C}$ foi de $5,66 \pm 0,17$. A constatação se estende para acidez, uma

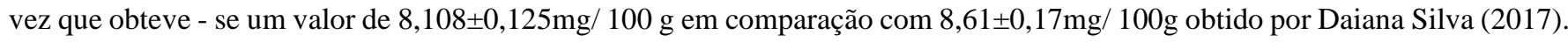
A legislação brasileira não estabelece limite máximo de acidez para farinhas de frutas; porém, a RDC n ${ }^{\circ} 12$ estabelece um limite máximo de 3\% de acidez para a farinha de trigo (Brasil, 1978). Portanto, observa-se que a farinha de polpa de baru processada no presente trabalho apresenta caráter ácido. Os níveis de acidez de um alimento podem ser resultantes de compostos naturais presentes no alimento (Fernandes et al, 2008). Alimentos ácidos apresentam vantagens em relação à conservação, uma vez que a acidificação inibe o crescimento de microrganismos (Pereda et al., 2005). Em relação às massas alimentícias, a legislação (Brasil, 2000) estabelece limite máximo para o parâmetro acidez, o valor de 5\% (5mg/ 100g). Portanto, observou-se que os macarrões com $100 \%$ de farinha de trigo e com $10 \%$ de farinha de polpa de baru atendem ao parâmetro da legislação vigente. Ainda, a mesma Resolução não estabelece limites para os valores de $\mathrm{pH}$. De acordo com a literatura, dependendo da natureza do alimento (composição química e pH), este pode ser classificado em alimentos ácidos ( $\mathrm{pH}<4,6)$ e aqueles de baixa acidez $(\mathrm{pH}>$ 4,6) (Aguirre \& Gasparino Filho, 2002). Assim, as massas alimentícias podem ser consideradas como alimentos de baixa acidez.

Por fim, notou-se a redução do valor energético à medida que substituiu - se a farinha de trigo pela farinha de polpa de baru. Tal redução é resultado do aumento de fibras nas formulações desenvolvidas (Pereda et al., 2005).

\subsection{Análises microbiológicas da farinha de polpa de baru e das massas alimentícias}

A partir das análises microbiológicas utilizando-se os parâmetros exigidos pela RDC n 12 (Brasil, 2001), obteve-se os resultados apresentados na Tabela 3. 
Tabela 3: Resultados das análises microbiológicas.

\begin{tabular}{|c|c|c|c|c|c|}
\hline Parâmetros & $\begin{array}{l}\text { Limite segundo } \\
\text { RDC } N^{\circ} 12\end{array}$ & FPB & FT & F1 & F2 \\
\hline $\begin{array}{l}\text { Bacillus cereus } \\
\text { (UFC/ g) }\end{array}$ & $3 \times 10^{3}$ & Ausente & Ausente & Ausente & Ausente \\
\hline $\begin{array}{c}\text { Coliformes a } 45^{\circ} \mathrm{C} \\
(\mathrm{NMP} / \mathrm{g})\end{array}$ & $10^{2}$ & Ausente & 1,000 & 3,000 & Ausente \\
\hline $\begin{array}{l}\text { Staphylococcus } \\
\text { aureus (UFC/g) }\end{array}$ & $\begin{array}{ll}\text { - } & \text { Não } \\
\text { especificado } \\
\text { para farinha } \\
\text { - } \quad \text { 5,00x } 10^{3} \text { para } \\
\text { massa } \\
\text { alimentícia }\end{array}$ & $1,00 \times 10^{2}$ & $1,00 \times 10^{2}$ & $1,00 \times 10^{2}$ & $1,00 \times 10^{2}$ \\
\hline $\begin{array}{c}\text { Salmonella sp } \\
\text { (Salmonella } \mathrm{sp} / \\
25 \mathrm{~g})\end{array}$ & Ausência & Ausente & Ausente & Ausente & Ausente \\
\hline
\end{tabular}

Legenda: FPB - farinha de polpa de baru; FT - amostra controle (100\% de farinha de trigo); F1 - amostra com $10 \%$ da farinha de polpa de baru; F2 - amostra com $20 \%$ da farinha de polpa de baru.

Fonte: Antunes et al.

Os resultados para Bacillus cereus sugerem que as matérias-primas, bem como os processos utilizados para a produção da farinha de polpa de baru e das massas alimentícias não apresentam riscos à saúde dos consumidores, pois não observou - se o desenvolvimento de tal patógeno. Em relação à Salmonella sp, tal microrganismo é encontrado no trato gastrointestinal do homem e dos animais; sua transmissão por alimentos, bem como as enfermidades causadas é de grande preocupação da saúde pública. Portanto, os resultados encontrados são um indicativo de que as condições higiênico-sanitárias em todo o processamento das massas alimentícias desenvolvidas foram adequadas (Cardoso \& Carvalho, 2006).

\subsection{Testes de qualidade das massas}

Os resultados para os testes de qualidade das massas podem ser observados na Tabela 4.

Tabela 4: Valores médios dos testes de qualidade das massas.

\begin{tabular}{cccc}
\hline Parâmetros & FT & F1 & F2 \\
\hline Tempo de cozimento (min) & $8,00 \pm 0,00^{\mathrm{a}}$ & $7,50 \pm 0,50^{\mathrm{b}}$ & $7,30 \pm 0,27^{\mathrm{b}}$ \\
Aumento da massa (\%) & $196,80 \pm 1,31^{\mathrm{a}}$ & $172,77 \pm 0,25^{\mathrm{b}}$ & $166,20 \pm 1,11^{\mathrm{c}}$ \\
Perda de sólidos $(\%)$ & $2,44 \pm 0,02^{\mathrm{a}}$ & $2,57 \pm 0,01^{\mathrm{b}}$ & $2,87 \pm 0,02^{\mathrm{c}}$ \\
\hline
\end{tabular}

a, b, c e d - médias seguidas de mesma letra não apresentam diferença significativa entre si pelo teste de Tukey a $5 \%$ de probabilidade.

Fonte: Antunes et al.

As amostras de massas alimentícias apresentaram tempo de cozimento variando entre 7 a 8 minutos. O menor tempo de cozimento foi observado para a massa preparada com $20 \%$ de farinha de polpa de baru. Portanto, percebeu-se que as substituições de 10 e $20 \%$ da farinha de trigo pela farinha de polpa de baru diminuiu em mais de $6 \%$ o tempo de cozimento das massas. Na literatura observa-se resultado contrário, pois com a substituição parcial da farinha de trigo pela farinha de mesocarpo de babaçu aumentou em mais de $11 \%$ o tempo de cozimento das massas (Neto et al., 2016). 
O teste de Tukey mostrou que não houve diferença significativa entre os tempos de cozimento das massas com $10 \mathrm{e}$ $20 \%$ de farinha de polpa de baru; porém, houve diferença significativa entre estas e a massa controle (100\% de farinha de trigo). Os resultados obtidos para o tempo de cozimento conferem às massas alimentícias com substituição parcial praticidade no preparo, atendendo a demanda dos consumidores.

Para o teste de aumento de massa, observou-se que a substituição parcial da farinha de trigo resultou na diminuição do aumento das massas alimentícias. Resultados semelhantes foram encontrados por Pietro (2016), em que a massa alimentícia com $100 \%$ de farinha de trigo apresentou aumento de massa equivalente a $213,53 \%$ e a massas alimentícias com 10 e $20 \%$ de farinha de polpa de baru, um aumento de $172,67 \%$ e $155,92 \%$, respectivamente.

Os resultados para o tempo de cozimento e o aumento da massa são justificados pela gelatinização do amido presente na farinha de trigo, pois é esperado que formulações com maior teor de farinha de trigo demorem mais para cozinhar e apresentem maior aumento de massa. A formação de gel a partir do amido envolve a mobilização de moléculas de água para dentro dos grânulos de amido, resultando em uma rede tridimensional que aprisiona tais moléculas. Logo, quanto maior a quantidade de amido na massa, mais tempo para cozinhar, maior aumento de massa devido ao aprisionamento das moléculas de água pelo gel e menor a perda de sólidos, uma vez que o amido está envolvido na formação do gel (Pietro, 2016; Cardoso \& Carvalho, 2006).

Em termos de perda de sólidos, observou-se que as massas alimentícias com substituição da farinha de trigo pela farinha de polpa de baru não apresentaram perda de sólidos superior a $6 \%$. As massas desenvolvidas no presente trabalho podem ser consideradas de muito boa qualidade, desde que macarrões nessa qualidade devem apresentar perda de sólidos em água de cozimento de até 6\% (Tomicki et al., 2015). Grandes perdas de sólidos são características indesejáveis e representa alta solubilidade do amido, resultando em turbidez na água de cozimento e baixa tolerância ao cozimento (Hummel, 1966; Bhattacharya et al., 1999).

\subsection{Análise sensorial das massas alimentícias}

A representação gráfica dos resultados do teste de comparação pareada entre a amostra controle (100\% da farinha de trigo) e as amostras com 10 e 20\% de farinha de polpa de baru, pode ser observada na Figura 3.

Figura 3: Representação gráfica do resultado do teste de comparação pareada.

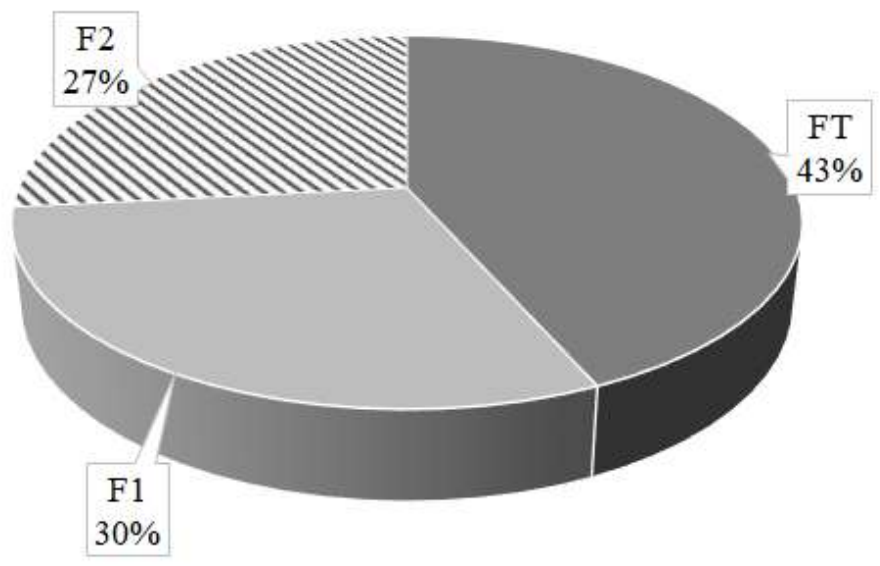

Legenda: FT - amostra controle (100\% de farinha de trigo); F1 - amostra com 10\% da farinha de polpa de baru; F2 - amostra com 20\% da farinha de polpa de baru.

Fonte: Antunes et al. 
O teste de comparação pareada entre os produtos demonstrou que as massas alimentícias com substituição parcial da farinha de trigo pela farinha de polpa de baru apresentaram preferência significativamente igual, para distribuição binomial de p > 0,05, à amostra controle (macarrão com 100\% de farinha de trigo).

Em relação ao teste de aceitação, pode observar-se na Figura 4 a média das notas atribuídas pelos julgadores às massas alimentícias desenvolvidas.

Figura 4: Representação gráfica do resultado do teste de aceitação.

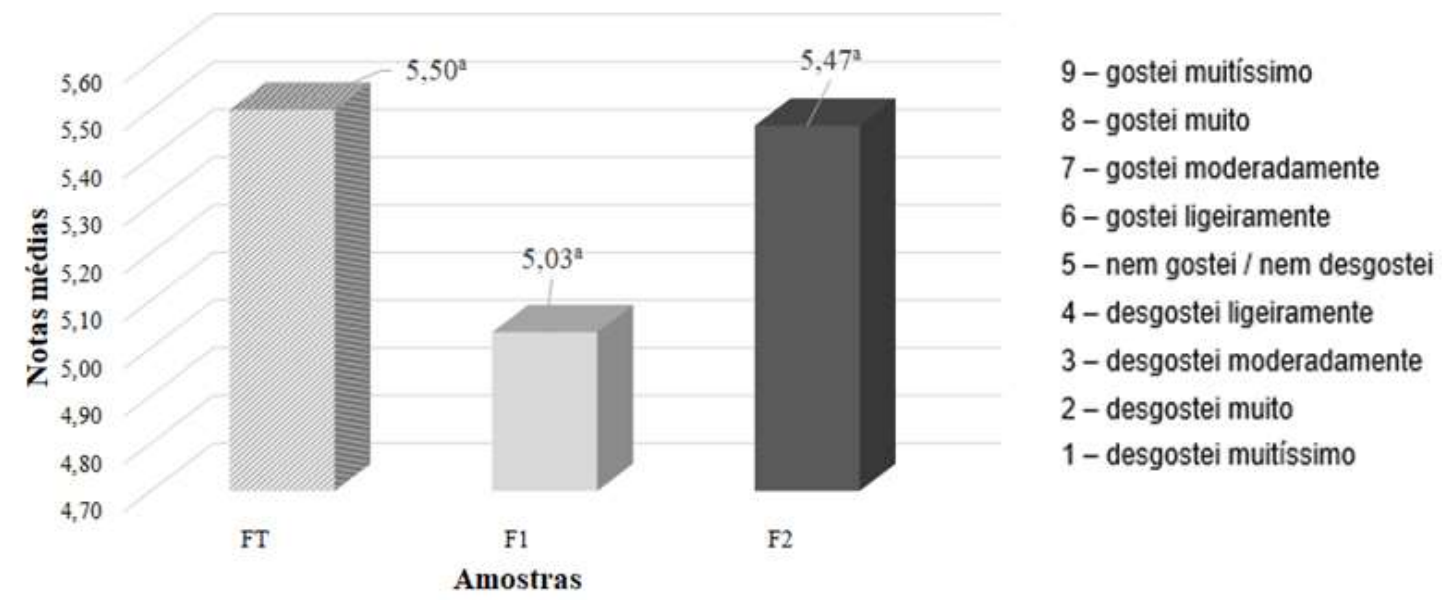

Legenda: FT - amostra controle (100\% de farinha de trigo); F1 - amostra com 10\% da farinha de polpa de baru; F2 - amostra com 20\% da farinha de polpa de baru. Médias seguidas de mesma letra não apresentam diferença significativa entre si pelo teste de Tukey a 5\% de probabilidade.

Fonte: Antunes et al.

Observou-se que as notas médias atribuídas pelos provadores não apresentaram diferença significativa entre si. Ainda, notou-se que todas as formulações apresentaram notas que variaram entre 5 - nem gostei/ nem desgostei e 6 - gostei ligeiramente. Foi relatado pelos provadores sabor residual amargo nas amostras F1 e F2; tal observação é resultante da presença residual de taninos na polpa, compostos fenólicos presentes em grande quantidade no baru in natura, mas que se perdem durante a estocagem (em função da maturação do fruto) e/ou são reduzidos nas etapas de sanitização e de aquecimento (Cruz et al., 2007; Ferrão et al., 2003). Ainda, foi observada a coloração das amostras, em que a amostra F2 apresentou coloração mais escura que F1; justifica-se a coloração mais escura pela alta quantidade de açúcares redutores apresentada pela polpa de baru, que quando aquecidos, resultam em escurecimento não enzimático do alimento (www.esalq.usp.br/departamentos/lan/pdf, recuperado em 01 de outubro, 2021).

Por fim, em relação ao teste de intenção de compra, pode observar-se na Figura 5 a média das notas atribuídas pelos julgadores às massas alimentícias desenvolvidas. 
Figura 5: Representação gráfica do resultado do teste de intenção de compra.

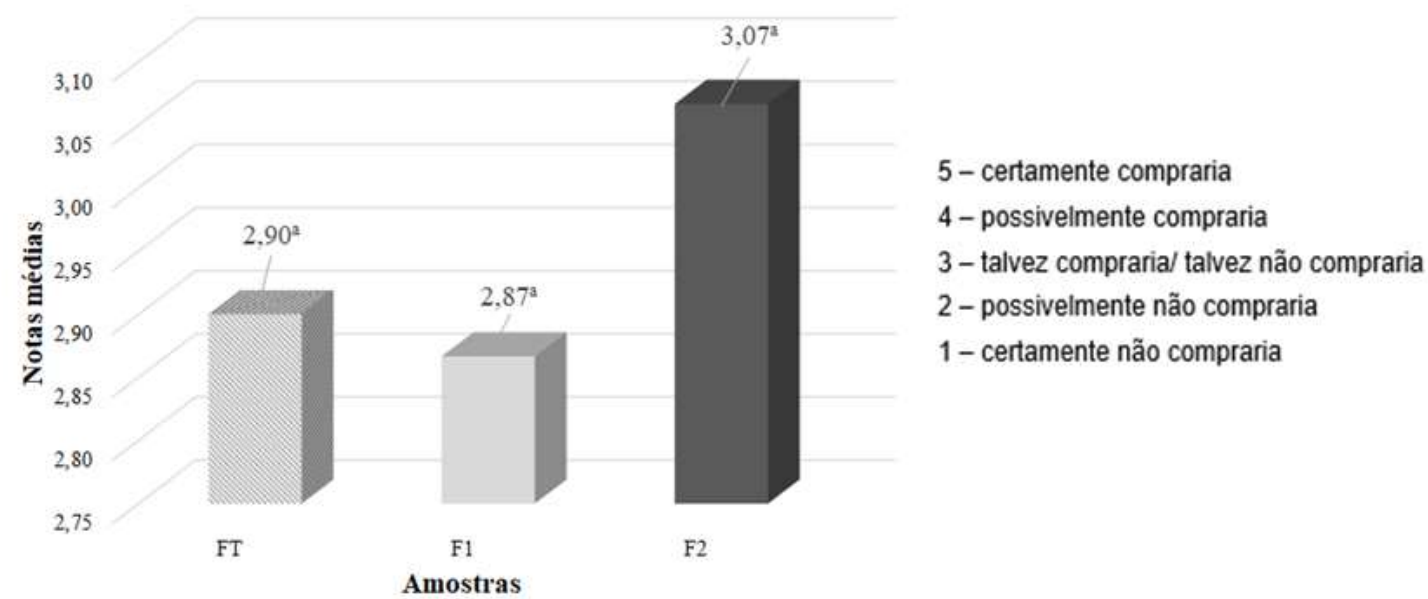

Legenda: FT - amostra controle (100\% de farinha de trigo); F1 - amostra com 10\% da farinha de polpa de baru; F2 - amostra com $20 \%$ da farinha de polpa de baru. Médias seguidas de mesma letra não apresentam diferença significativa entre si pelo teste de Tukey a 5\% de probabilidade.

Fonte: Antunes et al.

As notas médias apresentadas pelas amostras para o teste de intenção de compra não apresentaram diferença significativa entre si. As mesmas variaram entre 2 - possivelmente não compraria e 3 - talvez compraria/ talvez não compraria. Tais notas corroboram com os números apresentados no teste de aceitação.

\section{Conclusão}

A partir dos resultados apresentados, conclui-se que foi possível o desenvolvimento de um novo extrusado a partir da polpa de baru, uma vez que foram desenvolvidas massas alimentícias com substituição parcial de farinha de trigo pela farinha de polpa de baru; ainda, a partir da análise de fibras, observou-se que as mesmas massas alimentícias podem ser classificadas como alimentos com alto conteúdo de fibra alimentar.

Os resultados apresentados possibilitam a ampliação de aplicação da polpa de baru na indústria alimentícia e nos segmentos da agroindústria familiar (associações e cooperativas de produtores), uma vez que a amêndoa do fruto já é amplamente utilizada. Por fim, a essência da pesquisa foi integralmente alcançada, a partir dos resultados que mostraram o aproveitamento da massa residuária da polpa do baru no processo de extração da castanha.

Como considerações finais, ressalta-se como sugestão a possibilidade de melhoria das formulações desenvolvidas com farinha de polpa de baru, minimizando o sabor residual amargo apontado pelos julgadores na análise sensorial realizada. Para isso, sugere -se a neutralização das substâncias fenólicas aplicando-se extração da polpa com solvente ácido acético. Ainda, sugere - se também o desenvolvimento de formulações com diferentes porcentagens de farinha de polpa de baru. O aprimoramento na formulação dos macarrões possibilitará obter maior aceitação dos consumidores.

\section{Agradecimentos}

Aos Orientadores pelo direcionamento na execução da pesquisa; IFG Goiânia e SENAI Vila Canaã por disponibilizarem laboratórios e plantas didáticas para desenvolvimento das massas alimentícias e das análises.

\section{Referências}

AACC - American Association of Cereal Chemistry (1995a). Methods of the American Association of Cereal Chemists - Method 66-20 (8a ed.). Saint Paul: American Association of Cereal Chemists. 
AACC - American Association of Cereal Chemistry (1995b). Methods of the American Association of Cereal Chemists - Method 16-50 (8a ed.). Saint Paul: American Association of Cereal Chemists.

Aguirre, J. M. \& Gasparino Filho, J. (2002). Desidratação de frutas e hortaliças (206 p.). ITAL/FUNDEPAG.

AOAC - Association Of Official Analytical Chemists (1997). Official Methods of analysis of the Association of Official Analytical Chemists. Washington: Ed Washington.

APHA - American Public Health Association (2001). Compendium of Methods for the Microbiolo-gical Examination of Foods (4a ed.). American Public Health Association.

Atwater, W. O. \& Woods, C. (1986). The Chemical Composition of American Food Materials (v 28, n 47). Office of Experiment Stations.

Bhattacharya, K., Zee, S. Y., \& Corke, H. (1999). Physico chemical properties relates to qua-lity of rice noodles. Cereal Chemistry, 76 (6), 861-867. https://www.researchgate.net/publication/228605361_Physicochemical_Properties_Related_to_Quality_of_Rice_Noodles. 10.1094/CCHEM.1999.76.6.861

Brasil. Resolução CNNPA nº 12, de 21 de outubro de 1978. Normas Técnicas Especiais relativas a Alimentos e Bebidas. Brasília: ANVISA - Agência Nacional de Vigilância Sanitária. Recuperado de http://www.anvisa.gov.br/anvisalegis/resol/12_78_massas.htm

Brasil. Resolução RDC n. 93, 31 de outubro de 2000. Regulamento técnico para fixação de identidade e qualidade de massa alimentícia. Brasília: ANVISA Agência Nacional de Vigilância Sanitária. http://www.anvisa.gov.br/anvisalegis/resol/2000/93_00rdc.htm

Brasil. Resolução CNNPA n ${ }^{\circ}$ 12, de 02 de janeiro de 2001. Regulamento Técnico sobre padrões microbiológicos para alimentos. Brasília: ANVISA - Agência Nacional de Vigilância Sanitária. http://www.anvisa.gov.br/anvisalegis/resol/12_01_alimentos.htm

Brasil Resolução RDC n 360 de 23 de dezembro de 2003. Regulamento técnico sobre rotulagem nutricional de alimentos embalados. Brasília: ANVISA - Agência Nacional de Vigilância Sanitária. http://www.anvisa.gov.br/anvisalegis/resol/2000/93_00rdc.htm

Brasil Resolução RDC n ${ }^{\circ} 54$ de 12 de novembro de 2012. Regulamento técnico sobre informação nutricional complementar. Brasília: ANVISA - - Agência Nacional de Vigilância Sanitária. http://portal.anvisa.gov.br/documents/\%2033880/2568070/rdc0054_12_11_2012.pdf/c5ac23fd-974e-4f2c-9fbc-48f7e0a31864

Cardoso, T. G., \& Carvalho, V. M. (2006). Toxinfecção alimentar por Salmonella spp. Revista Instituto de Ciências da Saúde, 24 (2), 95 -101.

Carraza, L., \& Ávila, J. C. (2010). Manual Tecnológico de Aproveitamento Integral do Fruto do Baru. Brasília: Instituto Sociedade, População e Natureza.

Carrazza, L. R. (2010). Produção e comercialização de produtos agroextrativistas do Cerrado no PPP-Ecos: avanços, limites e desafios. In Lobo, A.; Figueiredo, I.; Andrade, K., Sementes lançadas, frutos colhidos: o Programa de Pequenos Projetos Ecossociais (p. 151). Brasília: ISPN.

Cavalcanti, R. B., Cardinot, G., Geotto, P., \& Pinheiro, R. S. (2012). Cerrado. In Scarano, F. R., Santos, I., Martins, A. C. I., Silva, J. M. C., Guimarães, A., \& Mittermeier, R. A., Biomas brasileiros: Retratos de um país rural (p. 304). Conservação Internacional.

Cruz, K. S., Silva, M. A., Freitas, O., \& Neves, V. A. (2011). Partial characterization of proteins from baru (Dipteryx alata Vog) seeds. Journal of the Science of Food and Agriculture, 91, 2006-2012. https://repositorio.unesp.br/handle/11449/7200. https://doi.org/10.1002/jsfa.4410

Cruz, S. E. S. B. S., Beelen, P. M. G., Silva, D. S., Pereira, W. E., Beelen, R., \& Beltrão, F. S. (2007). Caracterização dos taninos condensados das espécies maniçoba (Manihot pseudoglazovii), flor-de-seda (Calotropis procera), feijão-bravo (Capparis flexuosa, L.) e jureminha (Desmanthus virgatus). Arquivo Brasileiro de Medicina Veterinária e Zootecnia, 59 (4), 1038-1044. https://www.scielo.br/j/abmvz/a/rm9nyTk43 YrbTQf8vD8jvkP/?lang=pt. https://doi.org/10.1590/S0102-09352007000400033

Faria, E. V., \& Yotsuyanagi, K. (2002). Técnicas de análise sensorial (116 p.). ITAL/ LE-FISE.

Fernandes, A. F., Pereira, J.. Germani, R., \& Oiano-Neto, J. (2008). Efeito da substituição parcial da farinha de trigo por farinha de casca de batata (Solanum tuberosum Lineu). Ciência e Tecnologia de Alimentos, 28 (1), 56-65. https://www.scielo.br/j/cta/a/DRgtB5XtdM7XgnbWbczDjQn/?lang=pt. https://doi.org/10.1590/S0101-20612008000500010

Ferrão, M. F., Furtado, J. C., Neumann, L. G., Konzen, P. H. A., Morgano, M. A., Bragagnolo, N., \& Ferreira, M. M. C. (2003). Técnica não destrutiva de análise de tanino em café empregando espectroscopia no infravermelho e algoritmo genético. Tecnológica, 7 (1), 9-26. https://www.researchgate.net/publication/266567117_TECNICA_NAO_DESTRUTIVA_DE_ANALISE_DE_TANINO_EM_CAFE_EMPREGANDO_ESPE CTROSCOPIA_NO_INFRAVERMELHO_E_ALGORITMO_GENETICO.

Freitas, C. S., Antoniassi, R., Silva, T. S., \& Felberg, I. (2011). Coletânea de Métodos Analíticos para Determinação de Fibra (37 p.). Rio de Janeiro: Embrapa Agroindústria de Alimentos.

Guerreiro, L. (2006). Dossiê técnico. Rede de Tecnologia do Rio de Janeiro.

Hummel, C. (1966). Macaroni products: manufacture, processing and packing (287 p.). Food Trade.

IAL - Instituto Adolfo Lutz (2008). Métodos físico-químicos para análise de alimentos. Instituto Adolfo Lutz.

ISO - International Organization for Standardization (2002). Microbiology of food and animal feeding stuffs - Horizontal method for the enumera-tion of presumptive Bacillus cereus - Colony-count technique at $30^{\circ} \mathrm{C}-$ Method ISO 6579 . Switzerland: International Organization for Standardization.

ISO - International Organization for Standardization (2004). Microbiology of food and animal feeding stuffs - Horizontal method for the enumera-tion of presumptive Bacillus cereus - Colony-count technique at $30^{\circ} \mathrm{C}-$ Method ISO 7932. Switzerland: International Organization for Standardization. 
Klink, C. A., \& Machado, R. B. (2005). A conservação do Cerrado brasileiro. Megadiversidade, 1 (1). https://www.researchgate.net/publication/2283 42037_A_conservacao_do_Cerrado_brasileiro.

Marconi, M. A., \& Lakatos, E. M. (2017). Fundamentos de metodologia científica (8a ed.). São Paulo: Atlas.

Martins, B. A. (2010). Desenvolvimento tecnológico para o aprimoramento do processamento de polpa e amêndoa do baru (Dissertação de doutorado). Universidade Estadual de Campinas - UNICAMP, Campinas, São Paulo, SP, Brasil. http://repositorio.unicamp.br/jspui/handle/REPOSIP/256419.

Mendonça, A. L. (2008). Avaliação cinética de comportamento de componentes do baru (Dipteryx alata Vog.) para estudo da vida de prateleira da polpa do fruto (Dissertação de mestrado). Universidade Federal de Goiás - UFG, Goiânia, GO, Brasil.

Neto, A. A. C. (2012). Desenvolvimento de massa alimentícia mista de farinhas de trigo e mesocarpo de babaçu (Orbignya sp.) (Dissertação de Mestrado). Universidade Federal Rural do Rio de Janeiro - UFRJ, Seropédica, RJ, Brasil. https://tede.ufrrj.br/jspui/handle/jspui/1587.

Neto, A. A. C., Soares, J. P., Pereira, C. T. M., Gomes, M. S. S. O., \& Sabaa-Srur, A. U. O. (2016). Utilização de farinha de mesocarpo de babaçu de massa alimentícia fresca tipo talharim. Brazilian Journal of Food Research, 7 (1), 105-115. https://www.scielo.br/j/bjft/a/PVYp5jBgdjnVpm5rv VYfFFn/?lang=pt\#: :text=Brazilian\%20Journal\%20of\%20Food\%20Research,44\%25\%20no\%20teor\%20de\%20fibras. https://doi.org/10.1590/19816723.06318 .

Neto, U. B., Aguiar, J. P., Souza, F. C., \& Leitão, B. (2013, julho). Elaboração e caracterização do macarrão caseiro enriquecido com farinha de casca da pupunha (Bactris gasipaes KUNTH). Anais do Congresso de Iniciação Científica PIBIC/ CNPQ, Manaus, AM, Brasil, 2.

OMS - Organização Mundial da Saúde (2003). Manual das necessidades nutricionais humanas (69 p). Atheneu.

Pereda. J. A. O., Rodríguez, M. I. C., Álvarez, L. F., Sanz, M. L. G., Mingui-Llón, G. D. G. de F., Perales, L. de la. H., \& Cortecero, M. D. S. (2005). Tecnologia de Alimentos (v 1, 294 p). Artmed.

Pereira, G. S. L. [Gabriel], Braga, R. N., Souza, H. F., Oliveira, M. L. P., Lima, J. P., \& Vieira, C. R. (2017). Análises físico-químicas e tecnológicas em massa alimentícia fresca incorporada de farinha de coquinho-azedo (Butia capitata). Caderno de Ciências Agrárias, 9 (3), 1-5. https://periodicos.ufmg.br/index.php/ccaufmg/article/view/3002.

Pereira, A. S. [Adriana], Shitsuka, D. M., Parreira, F. J., \& Shitsuka, R. (2018). Metodologia da pesquisa científica (1a ed.). Santa Maria: NTE. https://repositorio.ufsm.br/bitstream/handle/1/15824/Lic_Computacao_Metodologia-Pesquisa-Cientifica.pdf?sequence=1 .

Pietro, A. S. (2016). Elaboração de massa alimentícia mista fresca tipo macarrão com substituição parcial da farinha de trigo por polpa de baru (Dipteryx alata Vog.) da região centro-sul de Mato Grosso e sua caracterização físico-química e potencial antioxidante (Dissertação de Mestrado). Instituto Federal de Mato Grosso Campus Cuiabá - IFMT, Cuiabá, MT, Brasil. Recuperado de http://ppgcta.ifmt.edu.br/wordpress/wp-content/uploads/2016/07/Pietro-A.-S.-_Elabora\%C3\%A7\%C3\%A3o-de-massa-aliment\%C3\%ADcia-mista-fresca-tipo-macarr\%C3\%A3o-com-substitui\%C3\%A7\%C3\%A3o-parcial-da-farinha-detrigo-por-polpa-de-baru-Dipteryx-alata-Vog.-da-regi\%C3\%A3o-centro-sul....pdf.

Rocha, L. S., \& Cardoso - Santiago, R. A. (2009). Implicações nutricionais e sensoriais da polpa e casca de baru (Dipteryx alata Vog.) na elaboração de pães. Ciência e Tecnologia de Alimentos, 29, 820-825. https://www.scielo.br/j/cta/a/syDM4vGyWrnXPNrTGTLsF7q/?lang=pt. doi: https://doi.org/10.1590/S010120612009000400019

Sano, S. M., Ribeiro, J. F., \& Brito, M. A. (2004). Baru: biologia e uso (52 p). Embrapa Recursos Genéticos e Biotecnologia.

Scariot, A., Souza-silva, J. C., \& Felfili, J. M. (2005). Cerrado: ecologia, biodiversidade e conservação (439 p). Ministério do Meio Ambiente.

Silva, D. V. [Daiana]. (2017). Propriedades nutricionais, físicas, químicas e tecnológicas funcionais das farinhas de baru (Dipteryx alata Vogel) em função de diferentes condições de secagem do fruto (Dissertação de Mestrado). Instituto Federal de Goiás Campus Rio Verde - IFG, Rio Verde, GO, Brasil. https://repositorio.ifgoiano.edu.br/handle/prefix/41.

Silva, R. F. da [Reginaldo], Ascheri, J. L. R., Pereira, R. G. F. A., \& Modesta, R. C. D. (2009). Aceitabilidade de biscoitos e bolos à base de arroz com café extrusados. Ciência e Tecnologia de Alimentos, 29 (4), 815-819. https://www.scielo.br/j/cta/a/mwVXHHdXgXLsQjgZTnfncRt/?lang=pt. doi: https://doi.org/10.1590/S0101 20612009000400018

Silva, D. B. [Dijalma], Silva, J. A. [José], Junqueira, N. T. V., \& Andrade, L. R. M. (2001). Frutos do Cerrado (178 p). EMBRAPA Informação Tecnológica.

Takemoto, E., Okada, I. A., Garbelotti, M. L., Tavares, M. A., \& Pimentel, S. (2001). Composição química da semente e do óleo de baru (Dipteryx alata Vog.) nativo do município de Pirenópolis, Estado de Goiás. Revista do Instituto Adolfo Lutz, 60 (2), 113-117. https://www.researchgate.net/publication/285313506_Composicao_quimica_da_semente_e_do_oleo_de_baru_Dipteryx_alata_Vog_nativo_do_Municipio_de _Pirenopolis_Estado_de_Goias.

Teixeira, E., Meinert, E., \& Barbetta, P. A. (1987). Análise sensorial de alimentos (180 p). UFSC.

Tomicki, L., Rigo, A., Durigon, A., Gutkoski, L. C., Zeni, J., Valduga, E., Steffens, C., \& toniazzo, G. (2015). Elaboração e avaliação da qualidade macarrão isento de glúten. Ciência Rural, 45 (7), 1311-1318. Recuperado de https://www.scielo.br/j/cr/a/RKvpPrgf3M59k5xhNLF4HMm/abstract/?lang=pt. https://doi.org/10.1590/0103-8478cr20140977

Vera, R., Junior, M. S. S., Naves, R. V., Souza, E. R. B., Fernandes, E. P., Caliari, M., \& Leandro, W. M. (2009). Características químicas de amêndoas de barueiros (Dipteryx alata Vog.) de ocorrência natural no cerrado do estado de Goiás, Brasil. Revista Brasileira de Fruticultura, 31 (1), 112-118. https://www.scielo.br/j/rbf/a/PnVntmr6Yw3zXJ5trGpxdNq/?lang=pt. doi: https://doi.org/10.1590/S0100-29452009000100017 